\title{
Proposal For A Management System For The Operation Of A Hybrid Network Platform Based On TDM Network Technology Migrating To Next-Generation NGN Networks
}

\author{
Ali Abdul Razzaq Taresh ${ }^{a}$, Noor Abdul Khaleq Zghair ${ }^{b}$ \\ ${ }^{a}$ Computer Engineering Department, University of Technology, Iraq. \\ E-mail:120005@uotechnology.edu.iq \\ ${ }^{\mathrm{b} C o m p u t e r ~ E n g i n e e r i n g ~ D e p a r t m e n t, ~ U n i v e r s i t y ~ o f ~ T e c h n o l o g y, ~ I r a q . ~}$ \\ E-mail: 120040@uotechnology.edu.iq
}

Article History: Received: 11 January 2021; Accepted: 27 February 2021; Published online: 5 April 2021

\begin{abstract}
Develop a proposal for a management model that allows, through knowledge management, to preserve the quality of services during the technological evolution of networks in a telecommunications company. Today, BaghTel is considered the main telecommunications company in Iraq and some time ago it was acquired by the Iraqi government, which has generated a significant organizational transformation, added to the fact of the rapid technological evolution and the strong competition of the sector. These factors give rise to concern on the part of certain units regarding which management model can best adapt to these changes. In addition to these reasons, there is a need for companies like BaghTel to improve the management of this type of updates or migrations, which would later translate into improvements in the quality of services provided by the company. This study seeks to propose a management model that allows optimizing the administration of networks and services, during the organizational transformation processes that occur in a telecommunications company as a result of today's technological evolution. The importance of this work lies in the opportunity to optimize, through a management model, the way to control an important technological change, which will ensure and improve the service levels that any telecommunications company provides to its end users. The final process, measures and assessments, include a viewing and use of management indicators of the results obtained in BaghTel's Specialist Networks Management; this consists essentially of a periodic monitoring and evaluation of the key variables in the management of the TDM (Time Division Multiplexing) and NGN (next generation of networks) networks;
\end{abstract}

Keywords: Next Generation of Networks, Time Division Multiplexing, BaghTel, Management Model.

\section{Introduction}

Currently, telephone companies worldwide are in different stages of the transition process to a next generation of networks based on IP (Internet Protocol), BaghTel (Baghdad Telecom) ${ }^{1}$, Iraq is no exception to this process. Telecom networks have recently undergone a great technological shift as a result of the first steps in the modernization of the traditional TDM (Time Division Multiplexing) next generation of networks (NGN). Companies migrating to NGN have to standardise operations and business processes, define new roles, train staff, negotiate relationships and set timetables according to the company's needs and commitments. Telecommunications companies have a desire to be constantly improving, and they are faster than their competitors $^{2}$. The project management unit requires difficult time from the operating units to meet, which could result in service failures, poor quality of service, and day-to-day operational problems in the services provided to end customers. Network services, by means of IP packets, are based on a technology that modifies basic access and communications parts in telecommunications networks. The most important change is the introduction of an IP layer independent of the network layer. From a managerial point of view, the new technologies and the need to acquire new knowledge on ICTs are common in companies that are immersed in this evolutionary process ${ }^{3}$.

All technological change has marked a fundamental change in organisations ${ }^{4}$. Organizations can only keep up by managing their internal processes well. In the BaghTel case study, there is a need to design a management model that ensures profit and transition with the least possible impact. There have been several methods of management: empowerment, intellectual capital, coaching, ISO standards, just in time, total quality management, etc.; they helped the organisation improve their products and services, and later provided greater customer satisfaction. Today, many telecommunication companies are switching to a packet switching type of network, known as a next generation network (Next Generation Network). Changes are generally made to reduce costs, both operation and capital. When making changes, changes usually are made quickly, causing a peak of work in the operations areas responsible for managing the network, possibly causing the quality of services offered to end customers. The networks of large telecommunications companies are made up of various technologies designed by different providers. This type of company usually has at least three large interrelated networks: a PSTN (Public Switched Telephone Network), an intelligent network, and the next generation network. Through these 
services, the company offers prepayment, presubscription, free calling, call with destination charge, nongeographic services. According to Thurow (2000), "knowledge has become the only source that guarantees a long-term competitive advantage". However, today in telecommunications companies like BaghTel, competitiveness can be measured apart from knowledge, depending on the contribution that companies make to society and their ability to adapt to changes. There have been several proposals to improve the way of working at BaghTel and to correctly manage the rapid technological evolution; despite this, the strong resistance to change in relation to paradigms oriented to information technology (IT) versus telecommunications technologies (Telco), time demands, high operational complexity and recent changes in society, may compromise quality of current and future services. The aim of this research work is, how to manage the technological innovation of networks, during the migration from a TDM network to NGN, in telecommunications operating companies, guaranteeing the quality of the services offered to end customers? And specifically, to identify the internal processes related to the management of the technological evolution of a telephony network and services platform, through a study that allows to reflect the information flow used by a technical support department in a telecommunications company, to explore the characteristics of the changes assumed due to the rapid implementation of new technologies in a telecommunications company and to identify how technological changes affect the operational development of a telecommunications company, compromising its evolution. This proposal is applicable to the telecommunications company BaghTel, since it is in constant technological evolution with a changing TDM and NGN hybrid network platform. The estimated time to carry it out is eight months. In the managerial aspect, previously with TDM technology, telecommunications companies such as BaghTel devoted time and effort to training their operational and managerial personnel in the different technical tools, turning this training into an important asset of collective knowledge, key to measuring production ${ }^{5}$. Today, with the transition to NGN technology, although there is a lot of technical training, it is observed that the knowledge in BaghTel is scattered with a focus on the individual, and is managed informally, this leads to a process of changes and intellectual construction by pieces (Telco's Vs. IT), which are responsible for day-to-day operations. In order to carry out this research, it was not possible to have the availability of all the necessary personnel, since there is a lot of resistance to technological change ${ }^{6}$.

\section{Methodological Framework}

Methodology used: The present work represents an investigation of the feasible project type. Said methodology refers to the development of a proposal for a viable model whose purpose is to satisfy a need or solve a problem. Feasible projects are those that are developed as an action to solve a particular situation, offering solutions in a methodological way. According to Arias, Fidias (2001) "a feasible project formulates action proposals and / or operating models as alternative solutions to the problem posed."

Level of Investigation: Currently many telecommunications companies have already started a process of migration from TDM networks to NGN such as BaghTel, however there are other companies in which this change represents only a vision for the future. The truth in both cases is that although there is a lot of documentation regarding technical architectures and best practices to manage platforms, there are operational problems and failures that affect customers during migration, a process that, due to the rapid technological implementation, has been little studied. In this sense, the level of the feasible project presented is considered an e xploratory type investigation, since according to Marciniak, (2008) ${ }^{7}$ "It is carried out when the objective is to examine a little-studied topic."

Research design: The design used in the feasible project refers to a combined documentary and field type research, initially it was necessary to study and analyze information regarding knowledge management in organizations that provide ICT services, PSTN and NGN networks, their operation and management, signalling protocols, and the migration process. Additionally, to know the operating environment of the networks installed in BaghTel, it was necessary to collect data, information and facts directly from reality, that is, in the Company, which in the same order of ideas as "Field research is one that consists of collecting data directly from the investigated subjects, or from the reality where the events occur (primary data), without manipulating or controlling any variable, that is, the researcher obtains the information, but not it alters the existing conditions" ${ }^{8}$.

Population and sample: This research covered the knowledge management functionality during the technological migration process from PSTN networks to NGN, the study environment of the research was the BaghTel Specialized Network Operations Management, the unit responsible among other functions of the implementation, operation and maintenance of PSTN and NGN networks. 
Techniques and instruments for data collection: To compile the data of the present investigation, the Internet facilities (Web pages), specialized publications, texts and recent magazines were used, in order to obtain updated information on the research area.

To find out how the PSTN and NGN networks are currently managed, open book interviews were conducted with consultants, specialists and technicians from BaghTel's Specialized Networks Operations Management.

\section{Management Proposal}

\section{PSTN Network Management Before Migration to NGN Networks}

The case study of the present investigation is BaghTel, since its foundation, until today, it has been characterized as a company with a very large technological platform, where various supplier brands make possible the wide variety of services that are offered to end customers. This diversity in terms of providers has created a robust and complex network to manage, requiring investment in management tools (hardware and / or software) that serve to try to have a little more centralized control of the elements that make up the PSTN network ${ }^{9}$. At BaghTel, several operating units use various proprietary tools to manage particular communication protocols such as MFC-R2 and SS7, the Company has specialists and technicians to correct situations in each network model, however, the diversity of technological brands, added to constant changes have increased the complexity and costs of managing both networks. Additionally, each operational management has its own internal processes, which probably makes it difficult to clearly observe the methodology that each area uses to deal with network and service management. BaghTel's Specialized Networks Management is responsible, among other activities, for managing the signalling of the TDM and NGN network elements. Signalling, as it could be observed in the theoretical framework, can be considered the nerve center of the different services provided by a telecommunications company, which makes it necessary for the personnel working in this area to have a minimum necessary knowledge that allows: to relate to the network elements that interact, the communication between them and their relationship with the services offered to end customers. ${ }^{10}$ Currently, this management has processes associated with how to offer technical support to the issue of signalling among the different elements that make up the network, among them, as a result of interviews with the personnel working in this management, the general process for provide technical support for signaling failures in the TDM network. Although the inputs to the process to provide signalling support come from other units in BaghTel, and that additionally there are several ways to record the incidents that occur daily; Internally, the Specialized Networks Management observes that there are regularly services whose operability was compromised, and a fault report related to said incident was not received, which is an indication of management deficiencies. In conclusion, network management is individual according to the resolution area, each area or unit has its own knowledge, tools, methodologies and processes to provide the necessary support to the eventualities that affect its platform on the network and compromise the operability of the services.

\section{Management During the Migration from PSTN to NGN Networks (Current Situation)}

In the beginning of BaghTel a process of migration to NGN networks. At that time, the Planning Management presented to the Company, the NGN project with a vision to: simplify the voice network, centralize control and applications, provide savings in maintenance and energy consumption and optimize operation and maintenance, among other benefits of the new technology, such as convergence with other IP networks ${ }^{11}$. To this day, the Company continues to replace many analogue exchanges with access gateways and Media Gateways interconnected with digital exchanges have been incorporated, in such a way as to packetize the traffic originated in exchanges with circuit-switched technology. In the same way as when there was only the TDM network in BaghTel, the management of each service is individual, where the Network Operations Center (COR) has continued to be the unit responsible for monitoring and following up on the resolution units of all those failures or situations that at an operational level affect any service. Through telephone contact, an email and / or a ticket on Nettrip, the COR documents the events that require support from specialists and / or technicians (who regularly provide their services in other managements). The Nettrip system contains statistics reported by analysts, and it usually documents the following:

- Ticket number assigned by BaghTel.

- Person who attends and reports the event.

- Description of the problem or failure of the service.

- Start time and duration time to solve the event.

- Description of the solution to the event.

- Name of the person responsible for the attention and closing of the ticket

- Whether or not the failure is attributable to BaghTel. 
Figure 1. represents an example of the current relationship between the PSTN and NGN networks, in it you can see the elements of each network model, which are used to refer to when documenting any incident.

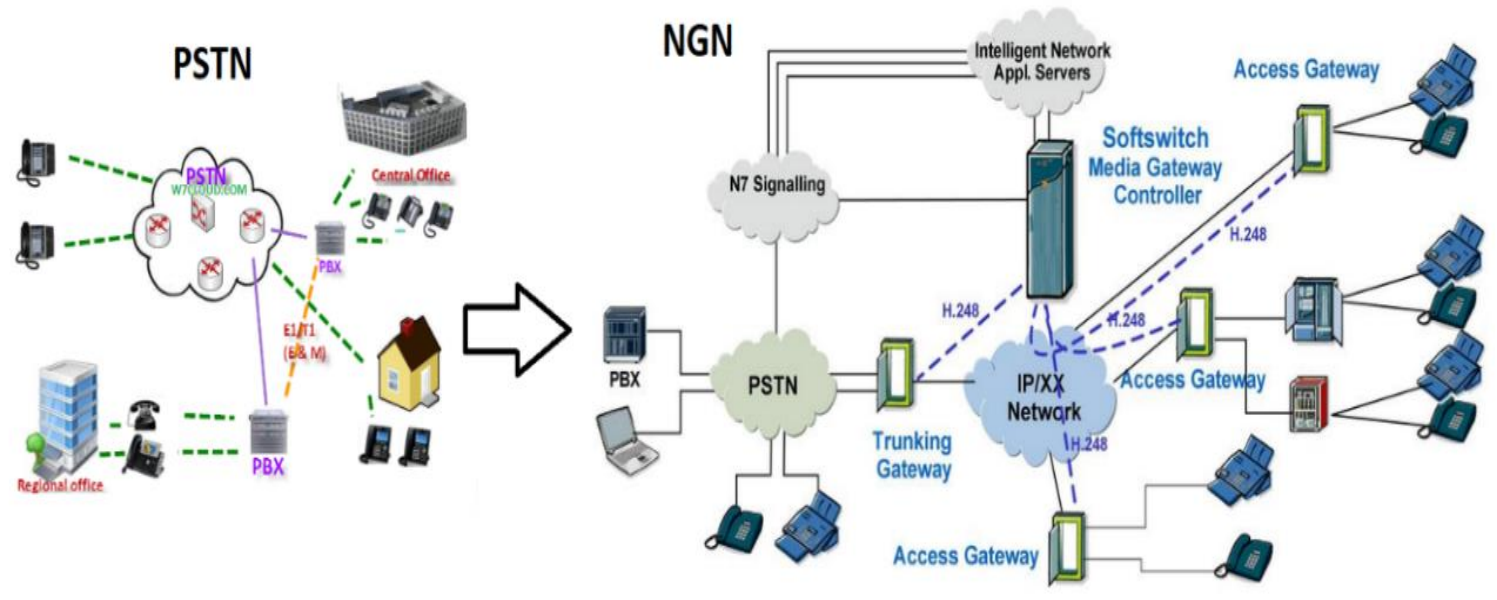

Figure 1. Current PSTN and NGN Network Architecture.

Currently, the Company continues to incorporate elements into the NGN network according to its growth plan, although in both networks there are various management systems, depending on the technology provider of each element. Having a management system for each technology provider makes BaghTel a company that requires a high investment in specialized human capital, both in the resolution area and in each brand (call it Huawei, ZTE, Nec, Alcatel, Siemens, Ericsson, HP, Nextone, among others), which creates a highly varied and complex work environment, full of diverse knowledge, especially for performing operations and maintenance tasks nationwide. In IT management terms, this is known as isolated technology pool management. ${ }^{12}$ BaghTel's Specialized Networks Management currently sees the efficiency in the management of networks and services compromised during the evolution project to NGN networks; basically between the factors of resistance to change (mentioned in the theoretical framework: people, culture, task, technology, design and strategy), which normally occur in any organization due to an important change, which This management is most concerned about the strategy to manage the knowledge related to the evolution of networks and services. All this situation previously exposed, together with the theoretical framework presented, serve as the basis for the present investigation; Without a doubt, the migration to NGN networks poses an important change in the way of managing the network platform, all the knowledge behind this project can represent for companies like BaghTel, a group of useful integration functionalities to improve or maintain the quality of services.

Proposal of a management model that allows, through knowledge management, to preserve the quality of services during the technological migration from TDM to NGN networks

BaghTel's current hybrid network platform is being managed, using individual process and technology groups. However, having well-designed and implemented processes is of little use if the day-to-day execution of these processes is not properly organised. There is no possibility of making improvements if operations data collection and daily performance measurement activities are not systematically conducted. The process of management of special networks at BaghTel is in an inception phase, in which the required characteristics and requirements needed to properly manage specialised networks are not yet clearly defined. In order to improve network management, the technical staff contacted managers, consultants, specialists and technicians to share knowledge and create a work team. The ability to communicate, network, and share knowledge reside within the organisation, which is cultivated by "People" and "Relationship" and the technical documents, reports, presentations, technical training centre (example: TTC - BaghTel) from the organisation. "Two scenarios related to the management of networks and services that occurred in BaghTel during the migration to NGN networks" are utilised as a "visualisation (in part) of the characteristics of the changes assumed due to the rapid implementation of new technologies". The "Company probably compromised the quality of services with its end customers", because knowledge management is a necessary technical role for a successful implementation of knowledge management.

Scenario 1: Management of Telephone Lines, from TDM to NGN Network Elements 
As TDM systems were first introduced, a set of data was stored at each physical switch. At each physical port the technicians were notified of changes in the system, and new facilities were eventually built with TDM technology. To implement the remote management, a central book was used, which contained information about the zones, exchanges and numbering plans of the line. This information was used with the tool called "central book", which then allowed to know the status of the line and perform any needed corrections (remotely or on site as the case may be). Here is an example (Table 1) of information from the central book.

Table 1. Example of Records in "Central Book" in BaghTel

\begin{tabular}{|c|c|c|c|c|c|c|c|}
\hline COD. & CCR & NAME OF & NUME & ATION & CAPAC. & KIND & PUT \\
\hline AREA & C32 & CENTRAL & Initial & LINES & & SERVICE & SERVICE \\
\hline 212 & 375 & LUIS HURTADO (D) & 412000 & 412399 & 4.000 & EWSD & $10-12-19$ \\
\hline 212 & 205 & WOODMAN (D) & 461000 & 465089 & 40.900 & $\mathrm{AX}$ & 09-10-19 \\
\hline ... & ... & & & & & $\ldots$ & $\ldots$ \\
\hline 241 & 410 & YELLOW FLOWER (D) & 868000 & 868901 & 9.020 & NEAX & $11-11-19$ \\
\hline ... & $\ldots$ & ...... & & & & $\ldots$ & \\
\hline 251 & $50 \mathrm{E}$ & CHIVACOA (D) & 873000 & 873499 & 5.000 & NEAX & 05-08-19 \\
\hline
\end{tabular}

In order to meet the needs of its customers, BaghTel implemented a special register with a database of customers whose telephone line would have undergone a change, in such a way that the information on the zone, central and numbering plan was correct at the time of carrying out the tasks. However, the knowledge of the personnel in the system is not updated, while the migration of the clients, which probably caused that at the time of carrying out management tasks, only a small group of people dispersed in different units, is trained to use the system management of the NGN network. When the above scenario occurred, the technicians who had knowledge about installing and maintaining phones in network, needed to acquire new knowledge about managing phones on the network, which required a relationship with the providers of NGN (at that time Huawei and ZTE), to jointly operate and maintain the already installed network. Technical incidents at BaghTel were compromised and the failure to effectively manage the situation and an insufficient training of personnel resulted in slower work spaces and unprepared personnel which is why the technical incidents would not have been well handled.

\section{Scenario 2: Management of Changes Related to the Routing of Telephone Traffic in TDM and NGN Networks}

Telephone traffic management coordination, responsible for configuring more than 5 years the routing rules for local calls, national long distance (LDN) and international long distance (L DI). This coordination continued to configure the rules for routing only in the TDM network, but it was another work team who tried to carry out this same configuration in the NGN network, specifically in the Soft switch. In the same way as SMS messaging, when using SMS Gateway you have to use the client code to match the number; however, when using the wireless gateway you have to use the group code to match the number. The team of professionals with both telecommunications and information technology training. After the TDM and NGN networks were brought up, it was discovered that problems with badly configured routing rules caused failures in service delivery to local, long distance and international calls. An example of this would be: 1) a call from a location where the NGN network is not present or 2) traffic from a certain serial of calls where it seems that the telephone exchanges keep trying to connect to each other. It shows how in the past, information technology organisations used ITIL (Information Technology Infrastructure Library) as a means of communication and shared knowledge and it is now possible to implement change in a more coordinated way. Organizations tend to start the implementation of ITIL with the management of problems and incidents in the operational areas. However, others tend to start with the management of disciplines. This might affect the organisation through processes of technology changes. Aligning services with IT services management requires a paradigm shift, which is initially proposed, because of the importance of technologies, processes and network management, the personnel working with technologies, processes and network management receive training on service management, using the best ITIL practises. Table 2 shows the most significant differences between traditional management versus management based on the ITIL best practices model.

Table 2. Differences in the Way of Managing IT Services.

\begin{tabular}{ll}
\hline Traditional Management Model & \multicolumn{1}{c}{ Model using ITIL } \\
\hline Focus on technology & Focus on processes \\
Firefighter role & Preventive actions \\
Reactive operating profile & Proactive operating profile \\
Insulation - silos & Integration of the entire organization
\end{tabular}




\begin{tabular}{ll} 
Informal processes & Processes according to best practices \\
IT perspective & Business outlook \\
Product oriented & Business oriented \\
\hline
\end{tabular}

Key problems when approaching knowledge management in BaghTel's Specialized Networks Management include the following:

- Staff are regularly engaged in day-to-day failures (firefighter role), overworked, with priority over urgent tasks, and probably do not have the time to document important settings or changes in a formal way.

- As there is no documentation on configurations, or recent changes, the operating personnel generally tend not to trust the data recorded in the platforms, so always before carrying out any work, they resort to different ways to search for related information and its approval.

- The services are generally not well documented, the documentation is sometimes incomplete or is not adapted to the different areas, and therefore in practice the information that resides in these documents is useless.

- The data on the configuration of networks and services is recorded in the elements that make up both networks (TDM and NGN), but subsequently there is no review or operational audit procedure, so the information available is generally out of date or incomplete.

- Resistance to change, probably personnel who are used to managing networks and services according to isolated procedures, may feel rejection before the possibility of a change where it is necessary: 1) share knowledge, 2) apply best practices such as those mentioned in the ITIL model, 3) accept that the figure of a knowledge manager is something normal, and 4) Get out of their "comfort zone".

To minimise these difficulties, where there is a wide variety of knowledge about different technologies (including TDM and NGN networks), it is suggested to have a Knowledge Manager, whose acronym in English (CKO) refers to "Chief Knowledge Officer". The Knowledge Manager or CKO is responsible for knowledge management, they also might be referred to as Knowledge Gatekeepers, Knowledge Producers, Intellectuals or Intellectual Asset Managers. In BaghTel the Knowledge Manager could represent an important strategic role in initiating, promoting and developing knowledge about networks and services, especially during the technological migration that represents the jump of networks TDM to NGN, and surely in the future from NGN to IMS.

The Knowledge Manager suggested to BaghTel, specifically to the Specialized Networks Management, to manage the technological migration from TDM to NGN networks could, according to the following characteristics:

- Entrepreneur: Refers to a professional with a base and / or experience in telecommunications or ICT, whose enthusiasm, initiative and knowledge are focused on developing new projects related to ICT networks and services, regularly the profile of this type of people is oriented to act in the face of novelty, adventure and risk, typical of any major technological change; they are visionaries who attract new ways of doing things, focusing on visible results.

- Environmentalist: It is an attitude aimed at strengthening explicit and tacit knowledge at all times, by creating social environments such as teamwork meetings, learning spaces and interpersonal relationships. They are regularly technical leaders, with the ability to integrate different knowledge, making use of best practices such as ITIL.

- Consultant: Refers to operational experience in the installation, implementation and administration of telecommunications networks and services; with the ability to listen to new ideas and to be consistent in applying them and adjusting them to a vision with a focus on knowledge management. You must be willing to allow others to play the leading role, it is understood that your role is to serve as an agent of change.

- Technologist: Able to understand which technologies favor the knowledge management process, at a managerial level he must have technical experience in ICT projects, as well as technical support in telecommunications networks and services, and he must favor the process of applying knowledge in prevention and solution of technical failures.

Appointing a Knowledge Manager or CKO, can be a good start for a knowledge management program at BaghTel, subsequently it is suggested to design an architecture through stages, which serves to stimulate the importance that the efficient administration of knowledge on management of knowledge could take networks and services, allowing the generation of greater capacities and greater sustainability of competitive advantages.

\section{The Proposed Model and its Stages}


To suggest a management model in the face of the change from TDM to NGN technology, using knowledge management, a detailed study of the theoretical framework presented was carried out, the presence of a role known as Knowledge Manager (CKO) was recommended and an analysis of two operational management scenarios in BAGHTEL, which allows identifying the need from a technological and organizational point of view of different stages, represented in figure 2 .

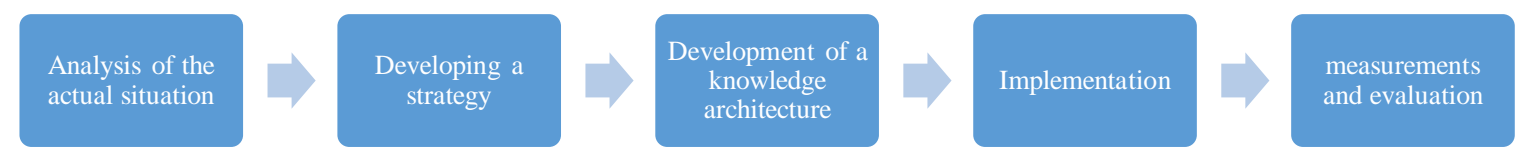

Figure 2. Structure of the Proposed Model

The initial stage refers to the need to analyse the current situation and the necessary knowledge in the face of technology changes (specifically from TDM to NGN), which play an important role in maintaining the quality of services offered to end customers.

\section{Analysis of the Current Situation}

The objective of this initial stage, with an approximate duration of one (1) month, is to suggest to BaghTel's Management of Specialized Networks, an analysis that allows understanding the importance of current technical knowledge as a significant role around the value of the Organization, Identifying the sources of knowledge, the best practices for network management, and the current and desired level of quality of service. For this, it is recommended to carry out at least 5 tasks:

1. Create the profile of the Knowledge Manager (CKO): Appoint her and identify the objectives and their scope within the Organization.

2. Establish a work team: Made up of a Knowledge Manager (CKO) responsible for coordinating the team and team agents, represented by responsible technicians and specialists.

3. Establish practical definitions: It is suggested to establish a clear definition of what BaghTel's Specialized Networks Management interprets as "knowledge". In certain managements this concept is related to experience, and in some others to capabilities. The importance of defining "knowledge" is to visualize the relationship that may exist with other concepts such as "value", "limits", "time window", "best practices", among others.

4. Identify the current strategy: It refers to an analysis that allows to know the levels of participation, and the interest of the staff in relation to the operational management of TDM and NGN networks, identifying the methodology and the current action plan to manage the different network elements and services.

5. Analysis of capacities: Within this analysis it is suggested to cover the technical and managerial capacities that the Management of Specialized Networks currently has to ensure the operational management of TDM and NGN networks in different scenarios.

\section{Development of a Knowledge Strategy}

This stage, with an approximate duration of one (1) month, consists of establishing a kind of bridge that will allow the Management of Specialized Networks of BAGHTEL, to go from where it is currently, to where it wants to be (in relation to maintaining the operation of services during the migration from PSTN to NGN networks), basically this stage aims to establish development plans that will allow, through knowledge management, to achieve the general objective of this research. For this, it is suggested to carry out at least 5 activities:

- Evaluation of core and secondary competencies: It is necessary to identify those core and secondary competencies necessary to efficiently manage the services and the network (TDM and NGN). Core competencies are those that have a high degree of participation, while secondary competencies, although they do not have a high degree of participation, may be related to some key knowledge factor.

- Analysis of knowledge gaps: Once the central and secondary competencies have been identified, it is advisable to establish the existing deficiencies on the sources of knowledge that support said competences, in this way it is possible to establish the current and desired level in relation to knowledge, This analysis will make it possible to establish the differences between what the Specialized Networks Management knows and should know in terms of managing TDM and NGN networks. 
- Definition of the goal and strategic objectives: The goal refers to the direction around which the actions should be aimed, it is suggested to develop the knowledge gaps identified above, and goal must be specific, measurable, consensual and realistic. Subsequently, groups can be defined by means of a work division structure, responsible for developing the objectives that will allow the goal to be reached, figure 3 represents an example:

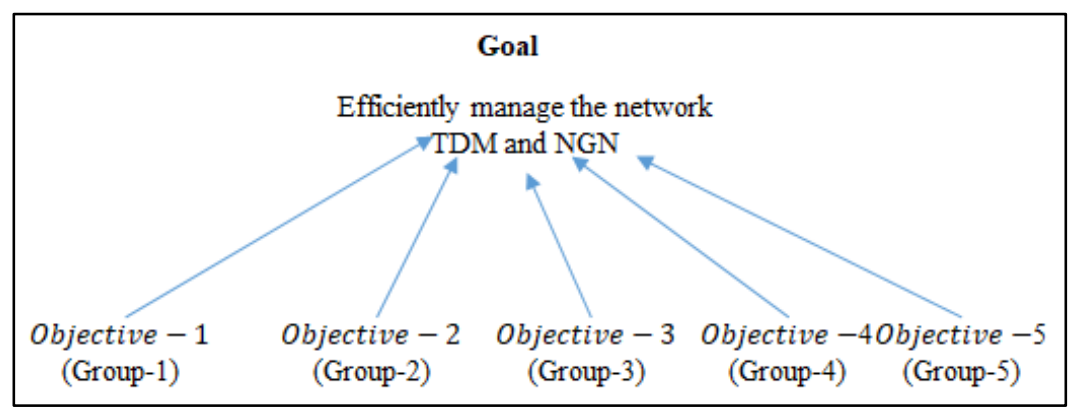

Figure 3. Structure of the Division of Objectives to Achieve a Goal

- Conversion of knowledge: Once the objectives that will make possible the goal "efficiently manage the TDM and NGN network" have been identified, it is through the interaction proposed by Huang, (2002) that they emphasize the dynamic model of the creation of the knowledge, where it is proposed that through the conversion of knowledge, the individual and technological requirements required by BaghTel's Specialized Networks Management can be defined.

- Development of short, medium and long term plans: It refers to the plans that broadly define the activities and actions necessary to give continuity to the goal of "efficiently managing the TDM and NGN network". In these plans, there could be the creation of another objective according to the need for knowledge, a result of the conversion mentioned above.

\section{Design of a Knowledge Architecture}

This stage lasts approximately 2 months, the purpose is to define the logical and technical basis on which the proposed management model will be developed, for this it is suggested to take into account the following:

- Requirements analysis: The objective of this analysis is to generate a specification of functional requirements on the environment and nature of the project, from a technological and social point of view. In this research, the technical environment is aimed at improving operational management during the migration of TDM networks to NGN, in the social environment through the use of ICT, the workers of the Specialized Networks Management will have the opportunity to reduce barriers and knowledge gaps in the operation and maintenance tasks of both networks, benefiting the society that makes use of the services offered by BaghTel.

- Investments in ICT: In the analysis of the current situation, it is necessary to identify the barriers that affect knowledge, in this sense, the investments suggested for ICT should ensure at least three aspects:

1. Reduction of spatial barriers: through the use of tools that make it possible to identify the stock of knowledge and inventory it, describing where it is located, people or work groups, if they exist in sources outside the Organization, as well as facilitating access to knowledge regardless of their physical location, including: corporate portals, virtual communities, videoconferencing, email and forums.

2. Reduction of temporary barriers: It is suggested to use tools that allow storing knowledge through chronological structures, facilitating simultaneous, structured and controlled access to knowledge, as well as communication and transmission of information in multiple formats in real time, in this sense, the use of: Data warehouse, multimedia systems, simulation software, intranets, email and forums is important.

3. Reduction of social barriers: These are those that facilitate formal and informal communication, temporary or continuous, regardless of hierarchical structures, among them the use of: virtual communities, Internet, Intranet, corporate portals and knowledge maps stand out.

- Hardware and software development and integration schemes: Refers to how BaghTel's Specialized Networks Management will establish the guidelines for developing applications (software) and acquisition of equipment (hardware) necessary for the knowledge management implementation process. 
- Technological analysis: This activity aims to determine those technologies that will support the knowledge management process (identification, selection of useful knowledge, structured storage, transfer and use of created and stored knowledge), for this it is suggested to the Management of Specialized Networks evaluate the existing technological options in the market taking into consideration five aspects:

1. Personalization: It refers to sharing knowledge through person-to-person contact. To facilitate this activity, you can use ICT such as portals, discussion groups, chats, Lotus Notes, among others.

2. Discovery: It is the activity of searching and obtaining knowledge from repositories and databases, for this it is suggested to use techniques of knowledge engineering, such as data mining (Data Mining) and text mining (Text Mining), among others.

3. Coding: It refers to the activity of capturing existing knowledge and placing it in repositories in a structured way, for this the use of techniques for knowledge acquisition (KA) is suggested.

4. Creation / innovation: Through this activity it is possible to generate new knowledge, the technology will be required to allow the use of the "Brainstorming" methodology or brainstorming, which consists of a conference technique, in which a group of people looks for the solution to a specific problem, gathering the ideas contributed by the participants.

5. Capture / monitoring: It refers to the process of capturing the knowledge transported in daily tasks as a result of the interaction between people and ICT, among them the use of useful tools for decisionmaking and knowledge portals can be highlighted, whose idea whether to measure the level of knowledge in a certain area, for example TDM and NGN networks, migration, management, signalling, among others.

Each of these activities can be aligned with the knowledge management process, in order to define an architecture of the management model by means of this relationship, (figure 4).

- Architecture of the knowledge management model: Represents the relationship of the activities mentioned above in the technological analysis and the knowledge management process.

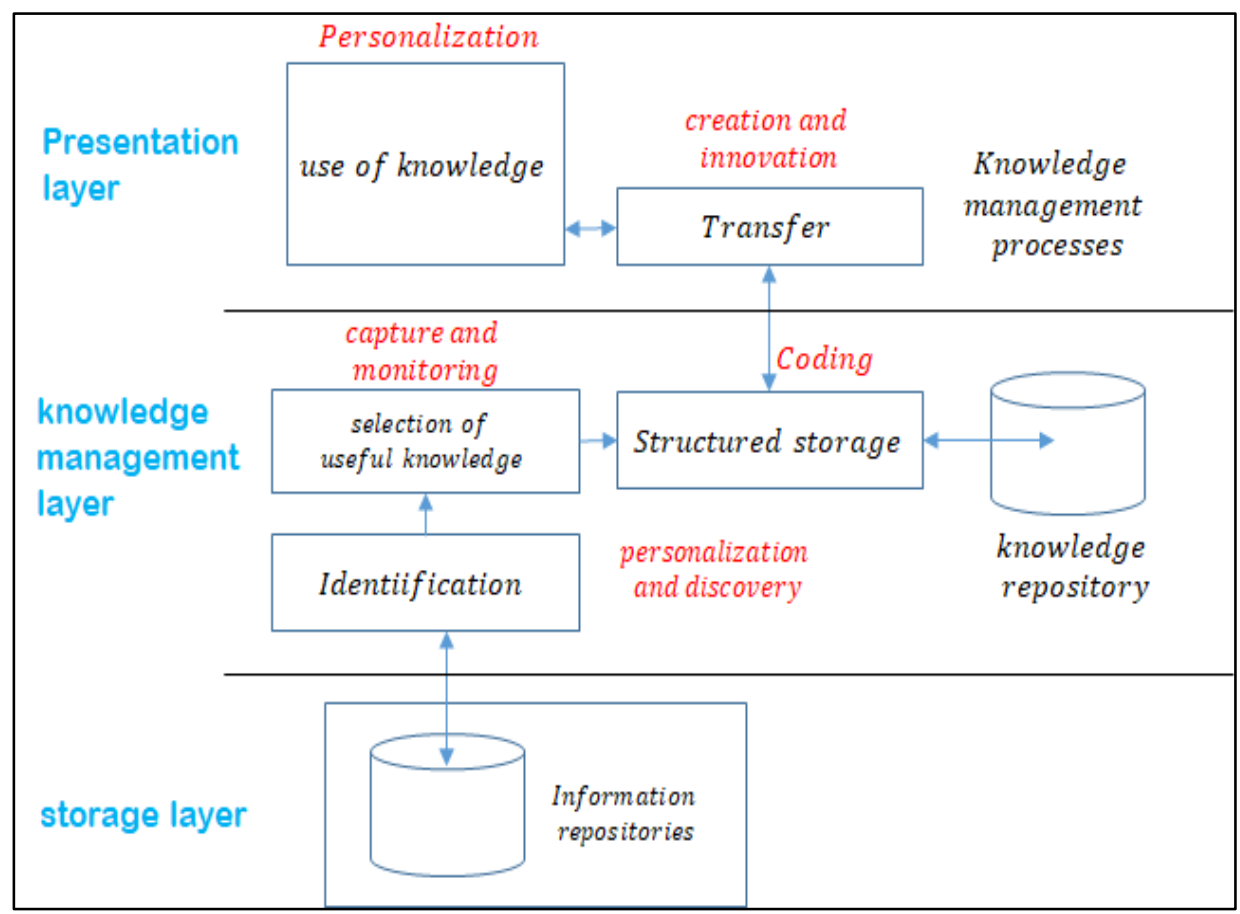

Figure 4. Proposed Architecture to Manage Knowledge

\section{Implementation}

The implementation phase, which lasts approximately two months, has as its main objective the development of the aforementioned activities, as well as establishing the basic guidelines for implementation. For this, four activities are suggested: 
1. Adaptation of the organizational structure: BaghTel's Specialized Networks Management represents the organizational structure of this research, in this sense, managerial and technical support is necessary in order to fulfil the goal "efficiently manage the TDM and NGN network", as well as the decision-making necessary for the implementation of the activities indicated in the knowledge strategy. As far as possible, it is suggested that one person be responsible for each objective and for the implementation of the strategies for their achievement.

2. Creation of the organizational climate: Within the implementation process, the creation of the organizational climate represents the most complex task, due to the resistance to change that will surely occur in the people who make up the organizational structure. To generate an organizational climate that supports knowledge management, it is suggested to communicate: 1) the expected benefits, 2) the objective, 3) the goal, and 4) the expected and obtained results.

3. Execution of activities: It refers to documenting and making known to all personnel the main characteristics of each activity in each of the objectives. It is important to reflect the origin, purpose and description of each activity, additionally, the capacity analysis must be taken into consideration. Each of the activities must be executed according to a planning hierarchy, that is, according to the detail established at the time of developing them.

4. Periodic review: Once the implementation of activities in each objective that lead to a final goal has been carried out, it is suggested to follow up through management measurements, the main idea is to visualize the results obtained with the incorporation of knowledge management

\section{Measurements and Evaluation}

The final stage, measurements and evaluation, consists of visualizing the results obtained in BaghTel's Specialized Networks Management with the implementation of knowledge management, with the use of management indicators; it basically consists of a periodic monitoring and evaluation of the key variables to manage the TDM and NGN network, below three evaluative categories:

1. Quantitative measurements: Describes measurements for the collection of data in conjunction with the tracking of the NETTRIP reporting system, showing how the information management (KM) system is being used, before, after and after its implementation.

2. Qualitative measurements: The breadth of the knowledge is evaluated by assessing the knowledge by analysing its quality by examining the score of all the inputs, the verbalization and the relative classification. Management workers feel comfortable with the network management by best practises and consistent tasks according to a specific goal.

3. Trained observers: Refers to certain measurements that employees trained in network and service management of the Specialized Network Management consider important to visualize.

\section{Conclusion}

Convergence represents a major technical evolution, but it is not about deploying new networks and services, but how to handle them to guarantee the correct activity of the same. Maintaining information in times of technological changes is an essential strategic advantage for organisations that provide these services. It needs a vision aimed at increasing the quality of service and the satisfaction of the customers. Answering the issue of how to handle the technical advancement of networks, during the migration from a TDM network to NGN in telecommunications operating companies, ensuring the quality of the services that are provided to customers. It means that technology migration processes must be closely controlled by the company that makes the transition, as well as knowledge management, without a doubt, contributes to improving the quality of decisions that need to be taken in every company, particularly in technical ones, where new knowledge is developed daily as a result of rapid evolution. The processes are managed by information management, which ensures the resources are used more effectively, work is not duplicated, and errors can be avoided. Businesses historically have relied on higher levels of management to formulate strategies. The problem is that ICT leaders are only now beginning to play a role in the region. IT knowledge complements best practises for management. Organizational growth, leadership, knowledge management, and others are essential to provide a body of knowledge to strengthen businesses. The key challenge of knowledge management is the lack of knowledge by many organisations with respect to the opportunity that an effective knowledge management model can create, and also the lack of interest in implementing a knowledge management method that has been shown to produce a profit. The challenge for professionals in information systems and ICT is the transition from information processing to knowledge processing; new technologies and strategies are required to facilitate the transformation of people from passive recipients of information to active agents contributing to the production of information and knowledge. 


\section{Recommendations}

- The implementation of a knowledge management model can provide BaghTel Specialized Networks Management with the key to efficiently manage the process of migration from TDM to NGN networks, guaranteeing the correct operation of services, as well as improvements in the process of decisionmaking necessary to promote growth and competitiveness, in this sense it is recommended to take into consideration the following aspects:

- Training is a fundamental aspect when you want to tackle a knowledge management project, so it is recommended to plan and structure it appropriately at different professional, technical, specialist, consultant, coordinator and managerial levels. Efficiently managing TDM and NGN networks is the key to success not only for the Management of Specialized Networks, but for the entire

- Setting realistic goals, the implementation of both knowledge management, represents a complex and cumbersome process that takes time; starting with four basic processes such as incident, problem, change and configuration management, can help define objectives, while facilitating resistance to change in relation to current network and service management.

- Delimit the activities of the proposed management model, with realistic times according to BaghTel's organizational culture, avoiding placing insufficiently prepared people in strategic positions.

\section{References}

1. http://www.baghtel.com/

2. Sharif, Sami. (2015). Migration to Next Generation IP Network. International Journal of Engineering and Computer Science. 4(1), 9916-9928.

3. Paudel, S. (2011). Migration of TDM network into NGN for the Fixed Wire-line access Network. In Second Asian Himalayas International Conference on Internet (AH-ICI), 1-5.

4. https://doi.org/10.1109/AHICI.2011.6113932

5. Alsaih, A.M., \& Almughaless, A.A. (2010). Next Generation Network design and capacity dimensioning. In International Conference on Educational and Network Technology, 561-566.

6. https://doi.org/10.1109/ICENT.2010.5532101

7. Almughaless, A.A., \& Alsaih, A.M. (2010). Optimum migration scenario from PSTN to NGN. In Second International Conference on Communication Systems, Networks and Applications, 1, $227-$ 231. https://doi.org/10.1109/ICCSNA.2010.5588698

8. Lundborg, M., Ruhle, E.O., Reichl, W., Ehrler, M., \& Wirsing, S. (2012). The migration to NGN from a regulatory perspective.

9. Marciniak, M. (2008). Future networks-beyond next generation networking. In 10th Anniversary International Conference on Transparent Optical Networks, 1, 25-28. https://doi.org/10.1109/ICTON.2008.4598361

10. Schneider, B. (2002). Network architectures: conversion strategies and evolution towards the nextgeneration network. Journal-Communications Network, 1(2), 47-51.

11. Liu, Jingxuan., \& Ansari, Nirwan. (2011). Public Switched Telephone Network. https://doi.org/10.1002/9781118256053.ch11

12. Medhi, D. (2007). Routing management in the PSTN and the internet: A historical perspective. Journal of Network and Systems Management, 15(4), 503-523. https://doi.org/10.1007/s10922-007-9085-6

13. Saeidi, M., Pirhadi, M., \& Ayazi, F. (2006). Intelligent network services in migration from PSTN toward NGN. In 8th International Conference Advanced Communication Technology, 1, 5.

14. https://doi.org/10.1109/ICACT.2006.205966

15. Yu, S.W., Tang, C.S., \& Lo, K.S. (2013). Transformation of PSTN to Next Generation Network. In 15th Asia-Pacific Network Operations and Management Symposium (APNOMS), 1-6.

16. Huang, J.C., \& Wang, S.F. (2002). Knowledge conversion abilities and knowledge creation and innovation: a new perspective on team composition. In Proceedings of the Third European Conference on Organizational Knowledge, Learning, and Capabilities, 5-6. 